'División de Políticas y Gestión de la Escuela de Salud Pública de la Facultad de Medicina de la Universidad de Chile, Santiago.

${ }^{2}$ Consejo Integrador de la Red Asistencial (CIRA) del Servicio de Salud de Bío Bío.

a Magíster en

Administración de Salud y Doctor en Salud Pública. bsicóloga Organizacional y Magister en Administración de Empresas.

cCirujano Dentista y Magíster en Salud Pública.

Recibido el 31 de mayo de 2012, aceptado el 7 de octubre de 2012

Correspondencia a: Marcos Vergara Escuela de Salud Pública, División de Políticas y Gestión, Independencia 939, Santiago

Teléfono móvil: 98216214 E-mail: mvergara@med. uchile.cl.

\title{
Competencias esenciales para la gestión en red
}

\author{
MARCOS VERGARA $^{1, \mathrm{a}}$, LIGIA BISAMA ${ }^{1, \mathrm{~b}}$, PATRICIO MONCADA $^{2, \mathrm{c}}$
}

\section{Essential competences for the management of health care networks}

We suggest that in order to fulfill the health needs of the majority of the Chilean population, which is beneficiary of the public health system, essential organizational skills should be developed for network administration among Self-administered Hospitals, Network Manager and Primary Health Care facilities. Self-administered Hospitals should be competent in managing service options according to their strategy for development, reference and counter-reference mechanisms and waiting lists, to optimize queuing. The Network Manager should be competent in demand management that is regulated, investments management that determines future development in terms of population needs and stakeholders' management, which is a political viability type of management. Finally, the Primary Health Care manager should be competent in demand management as a strategic partner of the Network Manager, community participation and management of interlinked areas, articulating social networks and sanitary impact management. At each level and within levels, there are crossroads that promote synergies. Based on the development of essential skills, a practice with strategic intentions, organization managers will develop team work skills.

(Rev Med Chile 2012; 140: 1606-1612).

Key words: Health services administration; Managed care programs; Public health.

L as competencias esenciales de una organización o core competences ${ }^{1}$ provienen del conjunto de conocimientos incorporados en aquellos ámbitos que le son propios y que están presentes tanto en el conjunto de la organización como en sus unidades conformantes. Ellas son las principales responsables de la generación de valor de cualquier organización.

La Escuela de Salud Pública de la Facultad de Medicina de la Universidad de Chile cuenta con una experiencia pionera de acompañamiento a directivos de servicios de salud, de hospitales autogestionados en red y de centros de atención primaria (APS), trabajando con equipos directivos para el desarrollo de competencias esenciales para la gestión en red en sus organizaciones públicas ${ }^{2}$.

Esta tarea resulta imprescindible para satisfacer una política de derechos garantizados a las personas $^{3}$ en un medio crecientemente competitivo y desafiante, que supone la propia operación de las Garantías Explícitas de Salud (GES).

Las organizaciones que gestionen bien sus competencias esenciales para la gestión en red habrán conseguido operar apropiadamente en el modelo asistencial cerrado con red única o prestador preferente para una población determinada, evitando la migración de sus usuarios en busca de alternativas normalmente más costosas, por encontrarse insatisfechos. Proponemos, entonces, que el buen funcionamiento de una red cerrada sea juzgado como tal en la medida que produzca satisfacción y confianza en sus usuarios.

Respecto a los modelos de gestión hospitalaria en red o gestión de la red asistencial, habría entonces competencias distintivas de la organización a desarrollar ${ }^{4,5}$, independientemente de que existan propuestas integradoras, como el Consejo de Integración de la Red Asistencial (CIRA). Proponemos 
que el aprendizaje de soporte para ello es el trabajo en equipo de sus directivos, logrado mediante una estabilidad mínima para comprometerse con esta visión compartida ${ }^{6}$.

Para representar esta propuesta, se utilizaron tres mapas estratégicos ${ }^{7}$ alineados ${ }^{8}$ entre sí. Tales mapas se muestran en las Figuras 1, 2 y 3.

\section{Hospital autogestionado en red}

Distinguimos tres competencias esenciales: desarrollar una cartera de servicios, consolidar un sistema de referencia y contra-referencia desde y hacia el hospital y gestionar las listas de espera (Figura 1) ${ }^{9}$.

\section{Desarrollar la cartera de servicios}

En el contexto de la autogestión hospitalaria la pregunta es ¿qué se produce aquí? (qué patologías organizan sus servicios y qué prestaciones y procedimientos clínicos desarrolla para ello) y ¿cuánto de aquello se produce aquí?, porque los volúmenes especializan ${ }^{10}$.

Esta tarea central y dinámica del hospital debe realizarse en función de los recursos disponibles y del desarrollo factible de las especialidades. Su frontera es la máxima capacidad resolutiva antes de pasar al nivel de atención siguiente y su piso es la máxima capacidad resolutiva del nivel anterior. $\mathrm{Su}$ composición -el mix de servicios- ha de estar en plena sintonía con las necesidades de la población (en parte reflejadas en las opciones de compra del asegurador o del derivador), debe ser explícito hacia el interior del hospital para dar una señal de ordenamiento de las funciones de producción y del uso de recursos asociado y debe generar compromisos de producción (metas) para medir resultados. Un hospital público que tiene

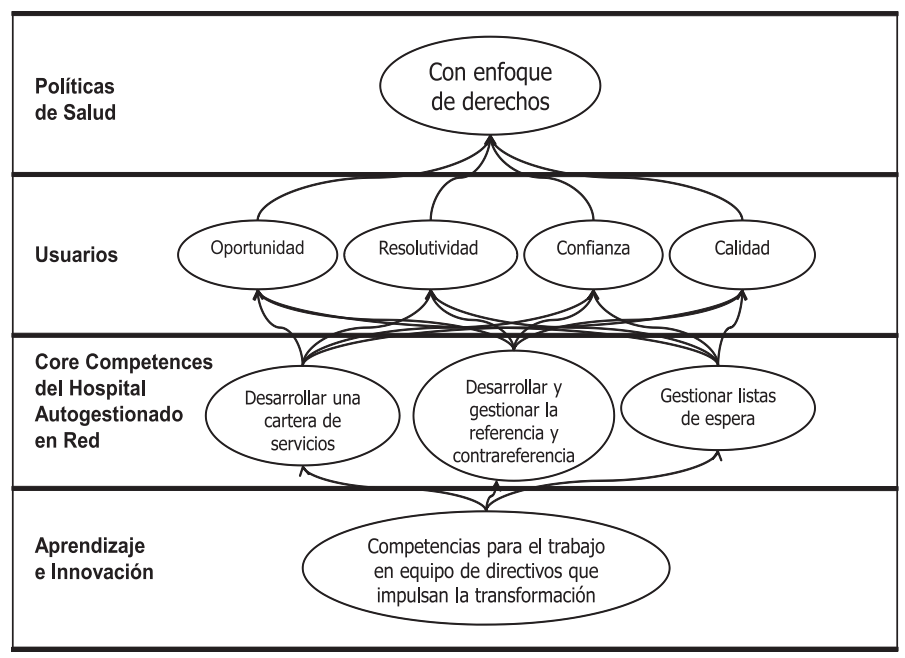

Figura 1. Mapa Estratégico Hospital en Red. una cartera de servicios definida y explícita y está orientado a su producción, es un hospital más gobernable y más transparente en su gestión.

Lo anterior, en todo caso, debe ser aprobado por el gestor de red, quien tiene la perspectiva epidemiológica de la población a cargo y, en el caso chileno, no debería estar exento de la contrastación de criterios con aquellos vigentes y consensuados en el CIRA.

\section{Gestión de la referencia y la contra- referencia}

Dimensión operacional y específica de la autogestión en red. Se debe definir, mantener y administrar la forma y condiciones en que los pacientes son derivados y contra-derivados a las especialidades para continuar sus tratamientos y controles en el origen, especificando cómo y con qué criterios son derivados a un nivel resolutivo mayor y bajo qué convenios de desempeño con el receptor. Esto es asunto de gran sensibilidad para pacientes y tratantes. De la expedición de estos mecanismos dependerá el juicio que las personas construirán respecto del sistema. Para el personal, impone una férrea disciplina respecto a la información, manejo de las agendas médicas, oportunidad de las altas, pertinencia en las derivaciones, formación y continuidad de los cuidados, poniendo en juego la performance de las "sinapsis" del sistema: cómo se conecta un nivel con el otro y cómo opera en la práctica la red.

\section{Gestión de las listas de espera}

Conocer y gestionar las listas de espera requiere orden, coordinación y retroalimentación entre distintos ámbitos del quehacer hospitalario. Implica contar con buenos registros, tener una sola lista actualizada, priorizar según criterios consensuados y explícitamente definidos y ofrecer la posibilidad de que las personas reali- 
cen seguimiento de su propia lista y sean atendidos en plazos razonables.

\section{El gestor de red}

Distinguimos otras tres competencias: gestionar la demanda, hacer gestión de stakeholders y gestionar el desarrollo de inversiones (Figura 2).

La gestión de la demanda supone una intervención anticipativa sobre la demanda y no una respuesta a la expresión libre y espontánea de la misma. Se basa en una lógica sanitaria y de costo-efectividad: promoción, prevención de enfermedad y de complicaciones, diagnóstico precoz, tratamiento oportuno y en el nivel de resolución apropiado, derivaciones pertinentes y expeditas, mejores alternativas terapéuticas, etc. Se trata de una suerte de modulación de la demanda en el contexto de una red asistencial que permita cerrar las brechas oferta-demanda sin recurrir única y exclusivamente al camino de incrementar la oferta.

Un buen ejemplo de esta idea es el caso del hospital concesionado de Alzira, Valencia ${ }^{11}$, que cambió radicalmente el tipo de gestión cuando su financiamiento público pasó del pago por prestaciones hospitalarias (clásico fee for service, al estilo privado, inicialmente considerado en la concesión) al pago per-cápita, luego de concesionar al gestor toda la red asistencial y no sólo el hospital, transfiriéndole el riesgo y, especialmente, logrando estimular la gestión de red o de demanda.

Esto explicaría por qué aquellos que condujeron el diseño de la reforma del sector de la salud en Chile durante el gobierno de Ricardo Lagos, incorporaron al modelo de autogestión hospitalaria -tal vez intuitivamente-, el concepto "en red".

La segunda competencia dice relación con la gestión de "stakeholders" 12 , que en el caso de la red pública se

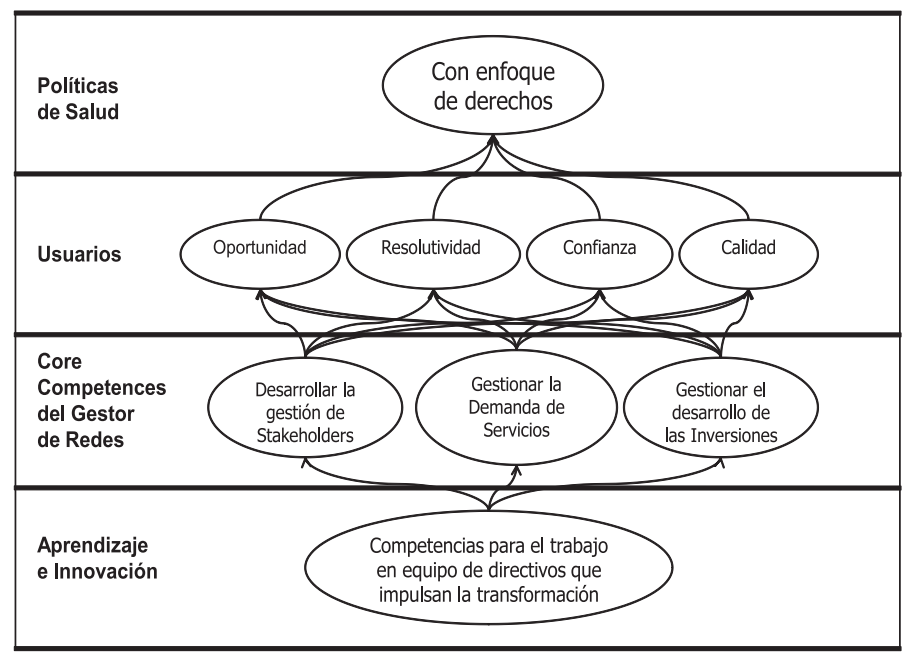

Figura 2. Mapa Estratégico Gestor de Red.

inserta en un panorama complejo por los variados problemas de agencia y gobernanza que suelen observarse y que son también problemas de gestión en sí mismos, pero estrechamente vinculados a la competencia esencial que aquí comentamos.

El dueño, quien se comporta como tal, o quien le representa, se difumina. La burocracia de turno (los gobiernos) han resistido históricamente las embestidas ciudadanas con argumentos de gobernabilidad, no obstante las razones de gobernanza han empezado a operar en el sentido contrario, al punto que la participación ciudadana en los asuntos públicos representa hoy un elemento central para su realización. Por otra parte, la línea de dirección o de gobierno suele ser rudimentaria (como es en el caso de Chile, con una línea de dependencia unipersonal) y el director de hospital aparece pendiendo de un hilo tan frágil para sostener sus decisiones como fuerte para arrastrar por el despeñadero a la autoridad de turno cuando se equivoca, pues en el propio seno del Ministerio de Salud está la sala de máquinas de comando-control de la red (en este sentido Chile ha retrocedido, porque incluso el Servicio Nacional de Salud -en los años 1950 y 60- tenía su propio comando, nombrado por el Parlamento, fuera del Ministerio).

$\mathrm{Al}$ interior se encuentran los gestores relacionándose ${ }^{13} \mathrm{con}$ sindicatos o asociaciones gremiales poderosas, estructuradas tras varias décadas de ejercicio del Estatuto Administrativo y otros cuerpos legales de semejante inspiración, como los que regulan la carrera médica y el nivel primario de atención. El Estatuto Administrativo ${ }^{14}$, de hecho, responde a un modelo concebido para el funcionamiento del Gobierno Central y no para empresas de servicio, a las que se asimilan mejor los hospitales.

En el caso de Chile, el origen de la demanda de los hospitales está en los centros de atención primaria, que operan 
mayoritariamente bajo la administración de los municipios, lo que despliega una variada gama de gestores públicos (los alcaldes) de distintos colores políticos y con diversos intereses y redes sociales estructuradas a su alrededor.

El panorama, en consecuencia, incluye actores del más diverso origen, quienes están situados alrededor o dentro en todos los niveles de la red asistencial cuyos intereses necesariamente deben ser administrados. Creemos que el gestor de red debiera facilitar el desempeño global de la red asistencial, visibilizando tanto las preocupaciones de los stakeholders principales ${ }^{15}$, como los indicadores que darán cuenta de su gestión.

Aunque el hospital autogestionado en red y la APS a su nivel han de hacer gestión de stakeholders, se plantea que, aunque sea una competencia organizacional de orden general, no es en estos casos una competencia esencial, como sí lo es para el gestor de la red, por su responsabilidad sobre los resultados sanitarios globales en la población de su territorio.

La tercera competencia alude a la gestión de las inversiones, que comprende decisiones que son determinantes del modelo de atención y de la producción de servicios futuros de la red asistencial, es decir, de su desarrollo sustentable.

Tratándose del desarrollo de una red cerrada de servicios que opera bajo restricciones presupuestarias, las decisiones de inversión han de tomarse en función de un modelo de atención o de oferta de servicios que promueva la costo-efectividad global. La oferta integrada de servicios de distinta capacidad resolutiva y niveles de complejidad supone desmaximizar a las partes para maximizar el todo. Esta especie de lugar común de la buena administración cobra especial sentido en el caso de las inversiones, que suelen ejecutarse muchas veces bajo la presión de intereses políticos o de desarrollo clínico de los especialistas que no necesariamente conducen a las decisiones más razonables ${ }^{16}$. Desgraciadamente, el sistema de salud está poblado de productos originados por malas decisiones, algunas probablemente motivadas por la ansiedad de directivos que conocen el comportamiento cíclico del financiamiento de las inversiones en el sector público de la salud que responde más a la política fiscal que a una planificada política de desarrollo del sector.

Por ello las decisiones de inversión que comprometen el desarrollo de la red o el modelo de ser- vicios no pueden radicar en los propios hospitales, ni deben realizarse sin consideración al desarrollo conjunto de toda la red. El gestor de red es quien debe tomar esas decisiones, no así las inversiones de reposición o de actualización tecnológica que, muy por el contrario, deberían tomarse descentralizadamente en cada establecimiento.

De este modo el cierre de las brechas de servicios se completa con una apropiada modulación de demanda por una parte y con la adaptación inteligente de la red asistencial a esas demandas moduladas, por otra. En esto último, la gestión de las inversiones es una pieza fundamental.

Gestionar en un panorama de estas características requiere de competencias consolidadas, tanto para la gestión de la demanda, como para desarrollar una relación "ganar-ganar" efectiva con los interesados en la gestión de la red y para asegurar un programa de inversiones que armonice con las necesidades de desarrollo futuro de la red ${ }^{17}$.

\section{Atención primaria de salud}

La APS ha sido considerada la puerta de entrada al sistema de salud. La profunda revisión que ha experimentado en los últimos años ha llevado a plantear un nuevo modelo de atención que, incorporando elementos de la salud familiar, por un lado y, por otro, la visión del sistema de salud como un conjunto de establecimientos configurados en una red sanitaria de resolutividad creciente, se ha dado en llamar "Modelo de Salud Integral con Enfoque Familiar y Comunitario". Dicho modelo está inspirado en directrices de la Oficina Panamericana Sanitaria ${ }^{18}$, cuyo rasgo principal y distintivo (relacionado íntimamente con su sistema de financiamiento capitado) es el tener una población a cargo. Postulamos que desarrollar competencias esenciales para la gestión de la APS con un sentido de red marcará fuertemente el desempeño del modelo.

Se escogieron 3 competencias esenciales: gestión de la demanda, gestión de la participación comunitaria y del intersector, y gestión del impacto sanitario (Figura 3). Esta proposición surge de la experiencia de trabajo con grupos de APS y se pone a disposición de los interesados para su debate y perfeccionamiento. Por lo pronto, cabe reconocer que existen correlaciones entre ellas y entre éstas con las de los otros niveles de resolutividad, pudiendo dar lugar a potenciales sinergias. 


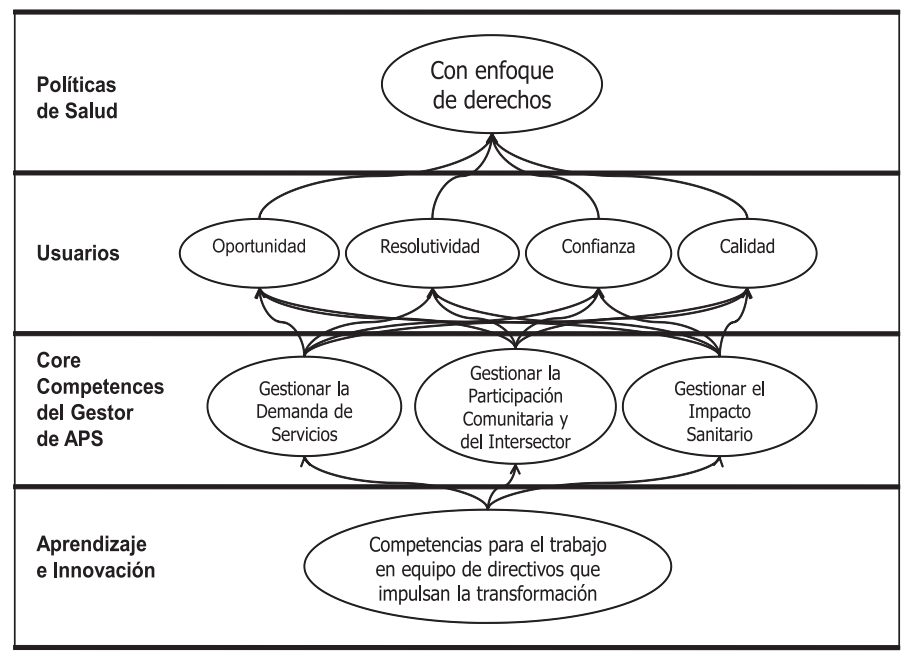

Figura 3. Mapa Estratégico APS en Red.

\section{Gestión de la demanda}

La gestión de la demanda supone una intervención anticipativa sobre la misma basada en una lógica sanitaria y de costo-efectividad y no sólo una respuesta a la expresión libre y espontánea de ésta: promoción de la salud, prevención de la enfermedad y de sus complicaciones, diagnóstico precoz, tratamiento oportuno y en el lugar apropiado (nivel de resolución), derivaciones pertinentes y expeditas, mejores alternativas terapéuticas, etc. Esto ya ha sido desarrollado al momento de referirnos a las competencias del gestor de la red. Sistémicamente es lo mismo, pero aplicado en un nivel de recursividad local, con la particularidad de estar en la primera línea de atención, y que debe aprovechar plenamente sus propios recursos para ello, generando sinergias con los demás actores de la red. Ambos, APS y el gestor de red, tienen población a cargo.

Un aspecto clave en este punto es cómo la APS es capaz de coadyuvar a gestionar una solución oportuna a aquello que excede su propia capacidad resolutiva: esta tarea involucra su mirada de red junto con la del Gestor de Red, pero también la dimensión operacional de la autogestión en red de los hospitales, esto es, la gestión de la referencia y la contrareferencia.

Los programas de resolutividad que permiten -por la vía de otorgar recursos frescos a los municipios- ir resolviendo las listas de espera para las especialidades con mayor demanda o falencia han creado una condición que representa una oportunidad para que los gestores de APS puedan mostrar sus habilidades negociadoras con otros niveles del sector público y con el sector privado, ampliando las fronteras de la ley ${ }^{19}$.

\section{La gestión de la participación comunitaria y del intersector}

El gestor de APS debe tener capacidad para establecer los procesos y mecanismos que le permitan escuchar a la comu- nidad y dar respuesta oportuna a los requerimientos de información y a la necesidad de conformar alianzas estratégicas con actores relevantes del ámbito sanitario como pueden ser los directivos del servicio de salud y de los hospitales o centros de referencia ambulatoria, los directivos de salud de otros municipios con quienes se pueden abordar problemas comunes, los actores del quehacer comunal como el alcalde, concejales, directivos municipales, gerentes o ejecutivos de otras agencias públicas, empresas e instituciones locales y de los medios de comunicación, dirigentes vecinales y comunitarios que frecuentemente opinan sobre la salud en la comuna, inclinando la balanza de la fe pública hacia uno u otro lado.

Que la organización cuente con capacidad anticipatoria y de articulación de intereses puede ser de notable ayuda al mejor desarrollo de su misión y contribuir a la percepción de respaldo que tengan los equipos de salud presentes en la comuna. La participación debe responder a un proceso ordenado de escucha, coordinación, compromisos y acción, apropiadamente gestionado gracias a las competencias específicas del equipo gestor de APS.

La participación debe producir en estos actores conciencia y acción de salud pública apoyando procesos que se dirijan a lograr mejores indicadores sanitarios comunales de acuerdo a una mirada macro relacionada con los objetivos sanitarios del país.

La participación es relevante porque aquí reside lo que pretende el modelo de atención: inclusión progresiva de la comunidad y del intersector en materias relacionadas con la salud comunal. Este aspecto es clave para equipos directivos que agregan valor público ${ }^{20}$. Valorar la opinión de aquellos hacia quienes dirigimos nuestros esfuerzos diarios por construir salud, así como de quienes pueden aportar 
lo suyo desde otras instituciones en pos de producir cambios positivos, generando mecanismos para incorporar esos aportes, debe necesariamente ser parte de las core-competences de los equipos directivos y gestores de APS municipal.

\section{Gestión del impacto sanitario}

Existe una amplia variedad de profesionales a cargo de salud municipal en las más de 300 comunas del país, no siempre con la debida formación en Salud Pública. Es evidente la tensión existente entre la presión asistencial y la posibilidad de desarrollar estrategias de anticipación a la aparición de los daños. Nuestros médicos, por lo demás, se sienten mucho más atraídos por estar en la trinchera -es de su naturaleza formativa- que por acompañar a los directivos en el desarrollo de programas de promoción y prevención. Es la APS el espacio para hacer la sintonía fina entre las políticas y la búsqueda de impacto sanitario país y las acciones concretas que es posible realizar para ello teniendo población a cargo.

\section{Recapitulación}

Hemos propuesto que para satisfacer las necesidades de salud de la amplia mayoría de la población chilena es necesario abordar el sistema de atención pública de salud en su conjunto, desarrollando competencias organizacionales esenciales para la gestión "en red" en los hospitales autogestionados en red, en el propio gestor de la red y en la Atención Primaria, con la perspectiva del sistema en su conjunto.

Las core competences que proponemos para cada uno de ellos son:

- Hospital autogestionado en red: i) Cartera de servicios; ii) Mecanismos de referencia y contra-referencia; iii) Gestión de las listas de espera.

- Gestor de red: i) Gestión de la demanda; ii) Gestión de la inversiones; iii) Gestión de stakeholders.

- Gestor de la APS: i) Gestión de la demanda; ii) Gestión de la participación comunitaria y del intersector y, iii) Gestión del impacto sanitario.

En cada nivel y entre niveles de la red existen cruces entre estas competencias, lo que permite suponer potenciales sinergias a explorar. El aprendizaje fundamental a la base de estas competencias esenciales, es la capacidad de trabajo en equipo de los directivos.

\section{Referencias}

1. Prahalad G, Hamel G. The Core Competence of the Corporation. Harvard Business Review, mayo-junio. 1990.

2. Vergara M, Bisama L. Autogestión Hospitalaria en Red II: El Método. Revista Chilena de Salud Pública 2010; 14: 52-8.

3. Cunill N. Las Políticas con Enfoque de Derechos y su Incidencia en la Institucionalidad Pública" Revista del CLAD Reforma y Democracia. Caracas. 2010; 46.

4. Le Boterf G. Ingeniería de las Competencias. Ediciones Gestión 2000 S.A.; Barcelona, 2001.

5. Montt J. Enfrentando las Transformaciones de los Hospitales Autogestionados. Capítulo III AUGE y Redes Asistenciales, páginas 133 a 150. "Reforma de la salud en Chile: desafíos de la implementación”. Sánchez H. \& Labbé J., Editores. Instituto de Políticas Públicas y Gestión Salud y Futuro. Universidad Andrés Bello. 2005.

6. Senge P. La Quinta Disciplina en la Práctica. Estrategias y Herramientas para construir la organización abierta al aprendizaje. Editorial Granica, Buenos Aires. 1995.

7. Kaplan R, Norton D. Mapas Estratégicos. Gestión 2000, Barcelona 2004.

8. Kaplan R, Norton D. Alignement. Gestión 2000, Barcelona 2005.

9. Ministerio de Salud. Establecimientos Autogestionados en Red (EAR). Plan de implantación de autogestión hospitalaria en red. Archivo Word. 2009.

10. Hax H, Majluf N. Estrategias para el Liderazgo Competitivo. Editorial Granica, Buenos Aires, Argentina, 2008.

11. European Observatory of Health Systems and Policies. Capital Invesments for Health. Case Studies from Europe. The Alzira Model: hospital de la Ribera, Valencia, Spain. Observatory Studies Series No18, Chapter 2. 2009.

12. Stakeholders Research Associates Canadá Inc., The Stakeholders Engagement Manual. Cobourg, Ontario, Canadá. 2005.

13. Flores F. Inventando la Empresa del Siglo XXI. Ediciones Pedagógicas chilenas S.A., 1989.

14. Ley 18.834. www.leychile.cl

15. Freeman RE, Harrison JS, Wicks AC, Parmar BL, de Colle S. Stakeholder Theory: The state of the art. Cambridge University Press. 2010.

16. Majluf N. Los Desafios de la Gestión. De lo formal a lo sutil. Aguilar Chilena Ediciones S.A. Santiago de Chile 
2011.

17. Albrecht $\mathrm{K}$, Albrecht S. Cómo Negociar con Éxito. El método de avanzada para construir tratos justos para todos. Coediciones Granica Vergara, Argentina, 1994.

18. Oficina Panamericana Sanitaria. La Renovación de la Atención Primaria de Salud en las Américas. Washington
D.C. 2007.

19. Musgrove P. Public and Private Roles in Health: Theory and Financing Patterns. World Bank discussion paper $n^{\circ}$ 339. 1996.

20. Moore MH. Gestión Estratégica y Creación de Valor Público, Capítulos 2 y 3. PAIDOS, Barcelona. 1998. 